\title{
NEFRECTOMIA DIREITA EM CÃO PARASITADO POR DIOCTOPHYME RENALE: RELATO DE CASO
}

\author{
L. G. SILVA ${ }^{1 *}$, M. T. IOZZI ${ }^{2}$, C. P. BURGÜER ${ }^{3}$, T. A. S. S. ROCHA ${ }^{4}$, A. C. CAMPLESI ${ }^{5}$, \\ M. B. CARVALHO ${ }^{6}$, A. E. SANTANA ${ }^{7}$, P. C. MORAES ${ }^{8}$
}

\section{RESUMO}

A dioctofimose, causada pelo nematódeo Dioctophyme renale, acomete frequentemente cães errantes e de hábitos alimentares pouco seletivos, podendo afetar também o homem. O parasita é grande, pode medir até cem centímetros de comprimento e se aloja geralmente no rim direito. O diagnóstico é obtido pela visualização dos ovos do $D$. renale durante o exame do sedimento urinário ou pelo achado do parasita à necropsia. O presente trabalho descreve um caso de parasitismo por este nematoide em cão atendido no Hospital Veterinário "Governador Laudo Natel" da Faculdade de Ciências Agrárias e Veterinárias, Jaboticabal, no qual a conduta terapêutica foi a realização de nefrectomia do rim acometido, devido à destruição do parênquima renal. A nefrectomia unilateral foi eficiente para a cura do paciente e eliminação do parasita.

PALAVRAS-CHAVE: Canina; Nefrologia; Verminose; Zoonose.

\section{SUMMARY}

Dioctophimosis, caused by the nematode Dioctophyme renale, frequently attacks erratic dogs and have poorly selective eating habits and, may also affects the man. The parasite is large, can be up to one meter long and usually is lodged to the right kidney. The diagnosis is obtained by the preview of $D$. renale eggs during the examination of the urinary sediment or by finding the parasite at necropsy. The present study describes a case of parasitism by this nematode in a dog attended at the "Governador Laudo Natel" Veterinary Hospital of the Faculdade de Ciências Agrárias e Veterinárias, Jaboticabal, in which the therapeutic management was the nephrectomy of the affected kidney, due to the destruction of the renal parenchyma. Unilateral nephrectomy was efficient for the cure of the patient and elimination of the parasite.

KEY-WORDS: Canine. Nephrology. Worms. Zoonosis.

\footnotetext{
${ }^{1}$ FCAV - UNESP - Campus de Jaboticabal. Email.: lari.g.silva@ hotmail.com

${ }^{2}$ FCAV - UNESP - Campus de Jaboticabal. Email.: mauricio_theago_iozzi@ hotmail.com

${ }^{3}$ FCAV - UNESP - Campus de Jaboticabal. Email.: ca_birg@ hotmail.com

${ }^{4}$ FCAV - UNESP - Campus de Jaboticabal. Email.: vetcraft3d@gmail.com

${ }^{5}$ FCAV - UNESP - Campus de Jaboticabal. Email.: annecamplesi@ yahoo.com.br

${ }^{6}$ FCAV - UNESP - Campus de Jaboticabal. Email.: mariledacarvalho@ gmail.com

${ }^{7}$ FCAV - UNESP - Campus de Jaboticabal. Email.: santana@ fcav.unesp.br

${ }^{8}$ FCAV - UNESP - Campus de Jaboticabal. Email.: pcastromoraes@ yahoo.com.br
} 


\section{INTRODUÇÃO}

A dioctofimose, causada pelo parasita Dioctophyme renale (D. renale), também conhecido como "verme gigante do rim", é o maior nematódeo conhecido chegando a medir de 14 a $100 \mathrm{~cm}$ de comprimento (BARRIGA, 2002). A doença apresenta distribuição mundial e trata-se de uma zoonose (VENKATRAJAIAH et al., 2014; PEDRASSANI; NASCIMENTO, 2015; NOROUZI et al., 2017). O parasito carece de especificidade de hospedeiro, e normalmente é descrito parasitando carnívoros domésticos e selvagens (ACHA; SZYFRES, 2003).

Seu ciclo de vida é considerado complexo, no qual ovos contendo larvas de primeiro estágio são ingeridos por um anelídeo oligoqueta aquático, que corresponde ao hospedeiro intermediário do $D$. renale. A infecção ocorre pela ingestão de anelídeos aquáticos, assim como de carne crua ou malcozida de peixe e rã contendo a larva infectante. $\mathrm{O}$ ciclo envolve hospedeiros paratênicos, como peixes e anfíbios. Dentre os vários hospedeiros definitivos, os carnívoros silvestres são os mais frequentemente parasitados, sendo relatado também em- felinos, suínos, equinos e bovinos (URQUHART et al., 1998; TRINDADE et al., 2018). Os cães são os principais hospedeiros definitivos para o $D$. renale quando se trata de espécies de animais domésticos. A disseminação da doença em ambiente urbano ocorre principalmente por cães de vida errante, os quais possuem hábitos alimentares pouco seletivos e, quando doentes, podem liberar os ovos pela urina (AMARAL et al., 2008; PERERA et al., 2017).

O D. renale tem predileção pelos rins, principalmente o rim direito, provavelmente devido à sua proximidade com o duodeno (PEREIRA et al., 2006). Entretanto, o parasita pode ser encontrado no rim esquerdo, em outros órgãos, livre na cavidade abdominal, e até invadindo o espaço intra-dural (BACH et al., 2016).

Geralmente os animais doentes são assintomáticos (URQUHART et al., 1998), porém, quando presentes, os sinais clínicos são inespecíficos e dependem da localização dos parasitas, podendo apresentar disúria, hematúria, dor lombar, desconforto generalizado, relutância em caminhar, poliúria e polidipsia, anorexia e êmese (AMARAL et al., 2008). $\mathrm{O}$ tratamento com uso de anti-helmínticos é contraindicado, uma vez que o parasito, devido a seu grande tamanho e espessura, dificilmente seria eliminado do rim (MEASURES, 2001).

Teve-se por objetivo relatar um caso de dioctofimose em cão atendido no Hospital Veterinário "Governador Laudo Natel" da Faculdade de Ciências Agrárias e Veterinárias / FCAV - Unesp Câmpus de Jaboticabal.

\section{RELATO DE CASO}

Um cão da raça Boxer, fêmea, de dois anos de idade, foi atendido no Hospital Veterinário "Governador Laudo Natel" da Faculdade de Ciências
Agrárias e Veterinária / FCAV - Unesp Câmpus de Jaboticabal. O tutor queixava-se que o paciente apresentava apatia, vômito, polidipsia e dificuldade de se manter em pé havia um mês. A paciente residia em uma chácara e possuía hábitos errantes.

Ao exame físico evidenciou-se linfonodo poplíteo esquerdo alterado e dor exacerbada à palpação lombar e abdominal. O exame neurológico foi realizado, observando-se ataxia proprioceptiva, com diminuição da propriocepção em membros torácicos e os reflexos patelar e flexor diminuídos em membros pélvicos, levando à suspeita de possível doença infecciosa devido à afecção dos quatro membros.

Exames complementares como hemograma e bioquímicos foram realizados, relevando-se anemia severa e discreta trombocitose (406.000). A concentração sérica de ureia $(33,7)$ encontrava-se normal, embora a creatinina $(1,69)$ estivesse discretamente aumentada. A urinálise de rotina também foi realizada permitindo a observação de cinco cruzes de hemácias, duas cruzes de leucócitos e inúmeros ovos do parasito $D$. renale. $\mathrm{O}$ paciente foi encaminhado para exame ultrassonográfico que revelou hipertrofia renal do rim direito, com arquitetura interna modificada e presença de várias estruturas arredondadas e tubulares, sugerindo a presença do $D$. renale, sendo então, encaminhado para procedimento cirúrgico.

Realizada tricotomia, com o paciente posicionado em decúbito dorsal e antissepsia local com uso de clorexidine degermante e alcóolico, realizou-se celiotomia pré-retro umbilical, seguida de localização do rim direito e nefrotomia para retirada dos parasitas, que consistiam em dois exemplares fêmeas (Figura 1). $\mathrm{O}$ rim direito apresentava comprometimento funcional devido à anormalidade anatômica observada ao ultrassom e durante o procedimento cirúrgico, optandose pela nefrectomia. O peritônio sobre o rim foi incisado, e com a ajuda de uma tesoura Metzembaum, realizou-se dissecção romba e cortante, liberando o rim de suas fixações sublombares. Identificados os ramos da artéria renal, uma dupla ligadura foi realizada com sutura monofilamentar absorvível (caprofyl 2-0) próximo a aorta abdominal, seguido da ligadura da veia renal de modo semelhante (FOSSUM, 2008).

À inspeção imediata macroscópica da peça cirúrgica observou-se destruição do parênquima renal com aspecto fibroso (Figura 2). O rim esquerdo também fora inspecionado, assim como demais órgãos da cavidade abdominal, não encontrando nenhuma outra lesão.

Ato contínuo, foi realizada a síntese do tecido muscular com sutura Sultan utilizando fio monofilamentar inabsorvível (nylon 2-0), seguido de aproximação do subcutâneo com sutura Zigue-zague e fio monofilamentar absorvível (caprofyl 2-0), concluindo com sutura de padrão simples interrompido na pele com nylon 3-0. As medicações pós-operatórias consistiram de enrofloxacina $(5 \mathrm{mg} / \mathrm{Kg}, \mathrm{q} .12 \mathrm{~h}, \mathrm{VO})$, ranitidina $(2,2 \mathrm{mg} / \mathrm{Kg}$, q. $12 \mathrm{~h}$, VO), meloxicam $(0,1$ $\mathrm{mg} / \mathrm{Kg}, \mathrm{q} .24 \mathrm{~h}, \mathrm{VO})$ e cloridrato de tramadol $(2 \mathrm{mg} / \mathrm{Kg}$, q.12h, VO). A ferida cirúrgica fora tratada diariamente 
por meio de limpeza com solução salina e clorexidine a $2 \%$, repetindo-se os curativos.

Com sete dias de pós-operatório, o paciente apresentava adequada reparação da ferida cirúrgica, e havia melhora em seu estado clínico, assim como normalização do exame neurológico. No décimo quinto dia de pós-operatório, realizou-se a retirada dos pontos e a paciente recebeu alta.

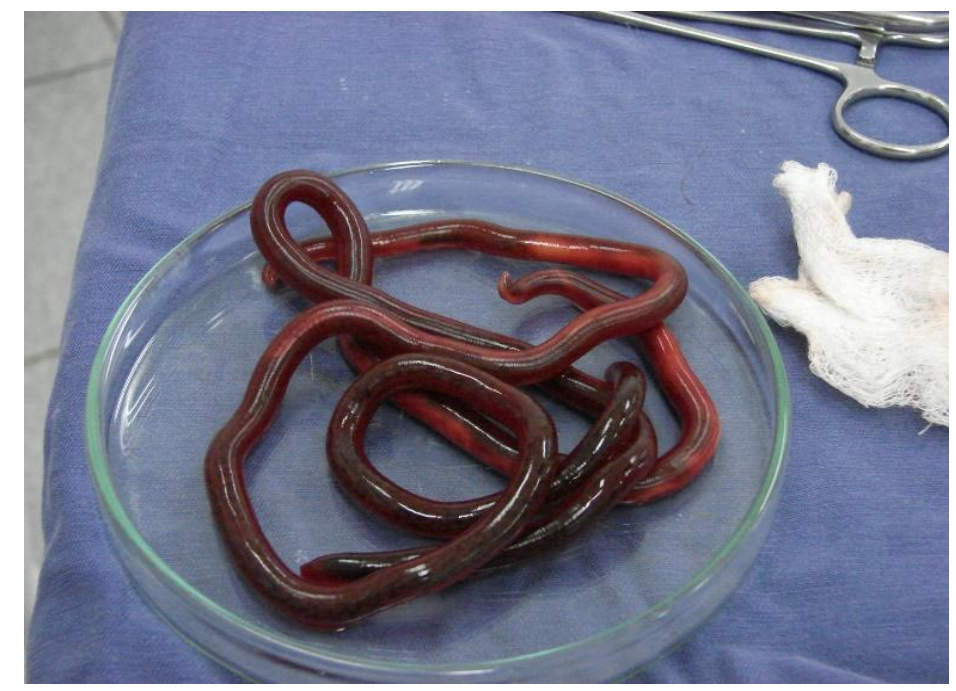

Figura 1 - Imagem fotográfica de dois exemplares de D. renale retirados do rim direito de cão por meio de nefrotomia.

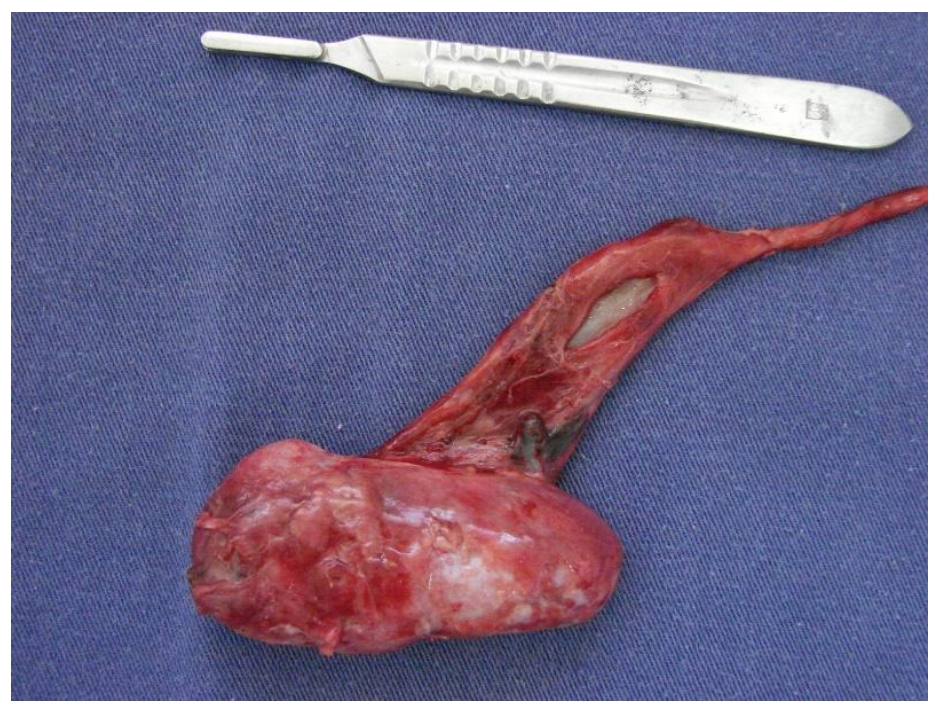

Figura 2 - Imagem fotográfica de rim direito de cão após nefrectomia. Notar aspecto fibroso e perda da anatomia normal.

\section{RESULTADOS E DISCUSSÃO}

A condição da paciente, a qual apresentava hábitos errantes e de deturpação alimentar, predispuseram-na à infestação pelo $D$. renale, assim como descreve Burgos et al. (2014) e Pereira et al. (2006). Geralmente, os animais parasitados são assintomáticos, contrariando os sinais clínicos observados no presente caso, tais como êmese, apatia, polidipsia, e dor lombar (URQUHART, 1998; AMARAL et al., 2008). É possível a presença de marcha cambaleante e distúrbios nervosos, justificando as alterações notadas no exame neurológico, como descrito por Pereira et al. (2006), devido à encefalopatia urêmica.
Em relação ao diagnóstico, exames radiográficos e ultrassonográficos dos rins e cavidade abdominal podem evidenciar a presença do parasita (LEITE et al., 2005). O exame do sedimento urinário, a fim de pesquisar a presença de ovos do parasita também se mostrou eficaz para a determinação do agente (LI et al., 2010), exame este que muitas vezes é negligenciado e sua importância subjugada (FOSSUM et al., 2008). Além de confirmar a presença do parasita no rim, o exame ultrassonográfico permitiu, também, a avaliação da estrutura anatômica renal (COTTAR et al., 2012). As discretas alterações encontradas no exame bioquímico justificam-se, pois segundo Amaral et al. (2008), em casos de acometimento unilateral do 
rim, não ocorrem aumentos de enzimas renais no sangue.

De acordo com Cottar et al. (2012), os animais parasitados pela forma adulta tendem a provocar destruição do parênquima renal, assim como fora detectado neste relato. Por vezes, é possível haver hipertrofia contralateral compensatória do rim sadio (LEITE et al., 2005; PEREIRA et al., 2006; NAKAGAWA et al., 2007), e quando alojados na cavidade abdominal podem causar peritonites crônicas, aderências e danos à superfície hepática.

Quanto ao tratamento instituído, a nefrectomia do rim acometido constitui-se imprescindível para cura da paciente (PERERA et al., 2017; SAPIN et al., 2017), uma vez que o parênquima do rim direito apresentava aspecto fibroso com perda da anatomia normal, assim como relatado por Trindade et al. (2018).

\section{CONCLUSÃO}

Com base na evolução do caso, verificou-se que a nefrectomia foi eficaz para cura definitiva da dioctofimose no cão do presente relato. Também, o caso coloca em evidência a importância dos cuidados com a higiene e alimentação dos caninos e do homem, pois trata-se de um problema de saúde pública, que deve ser incluído na lista de diagnósticos diferenciais.

\section{REFERÊNCIAS}

ACHA, P. N.; SZYFRES, B. Zoonoses and communicable diseases common to man and animals. Pan American Health Org. p.544, 2003.

AMARAL, L. C. D.; POLIZER, K. A.; SANT'ANA, T. M.; NEVES, M. F. Dioctophyma renale. Revista Científica Eletrônica de Medicina Veterinária Periódicos Semestral. p.10, 2008.

BACH F. S., KLAUMANN P. R.; MONTIANIFERREIRA F. Paraparesis secondary to erratic migration of Dioctophyma renale in a dog. Ciência Rural. v.46 p.885-888, 2016.

BARRIGA, O. Las enfermidades parasitarias de los animales domésticos em la America Latina. Santiago: Editorial Germinal. p.247, 2002.

BURGOS, L.; ACOSTA, R. M.; FONROUGE, R. D.; ARCHELLI, S. M.; GAMBOA, M. I.; LINZITTO, O. R.; LINZITTO, J. P.; OSEN, B. A.; RADMAN, N. E. Prevalence of a zoonotic parasite, Dioctophyma renale (Goeze, 1782), among male canines in a wild riverside area of La Plata River, Province of Buenos Aires, Republic of Argentina. Revista de Patologia Tropical. v.4 p.420-426, 2014.

COTTAR, B. H.; DITTRICH, G.; FERREIRA, A. A.; CARVAlHO, A. C. P.; ALBERNAZ, V. G. P.; LUZ, M. T.; TASQUETI, U. I. Achados ultrassonográficos de cães parasitados por Dioctophyma renale: estudo retrospectivo. Veterinária e Zootecnia. v.19 p.08-11, 2012.
FOSSUM, T. W.; DUPREY, L. P.; O'CONNOR, D. Cirurgia do Rim e do Ureter. In: FOSSUM, T. W. Cirurgia de Pequenos Animais. Elsevier: Brasil. 3 ed. 2008.

LEITE, L. C.; CÍRIO, S. M.; DINIZ, J. M. F.; LUZ, E.; NAVARRO-SILVA, M. A.; SILVA, A. W. C.; LEITE, S. C.; ZADOROSNEI, A. C.; MUSIAT, K. C.; VERONESI, E. M.; PEREIRA, C. C. Lesões anatomopatológicas presentes na infeção por Dioctophyma renal (GOEZE, 1782) em cães domésticos (CANIS FAMILIARIS, LINNAEUS, 1758). Archives of Veterinary Science. v.10 p.95$101,2005$.

LI, G.; LIU, C.; LI, F.; ZHOU, M.; LIU, X.; NIU, Y. Fatal bilateral dioctophymatosis. The Journal of Parasitology. v.96 p.1152-1154, 2010.

MEASURES, L. N. (2001). Dioctophymatosis. In: SAMUEL, W. M; PYBUS, M. J; KOCAN, A. A. Parasitic Diseases of Wild Mammals. Iowa State University Press: USA. 2 ed, 2001.

NAKAGAWA, T. L. D. R.; BRACARENSE, A. P. F. R. L.; REIS, A. C. F.; YAMAMURA, M. H.; HEADLEY, S. A. Giant kidney worm (Dioctophyma renale) infections in dogs from northern Paraná, Brazil. Veterinary Parasitology. v.145 p.366-370, 2007.

NOROUZI, R.; MANOCHEHRI, A.; HANIFI, M. A case report of human infection with Dioctophyma renale from Iran. Urology Journal. v.14 p.3043-3045, 2017.

PEDRASSANI, D; NASCIMENTO A. A. 2015. Verme gigante renal. Revista Portuguesa de Ciências Veterinárias. v.110 p.30-37, 2015.

PEREIRA, B. J.; GIRARDELLI, G. L.; TRIVILIN, L. O.; LIMA, V. R.; NUNES, L. C.; MARTINS, I. V. F. Ocorrência de dioctofimose em cães do município de Cachoeiro do Itapemirim, Espírito santo, Brasil, no período de maio a dezembro de 2004. Revista Brasileira de Parasitologia Veterinária. v.15 p.123$125,2006$.

PERERA, S. C.; RAPPETI, J. C. S.; MILECH, V.; BRAGA, F. A.; CAVALCANTI, G. A. O.; NAKASU, C. C.; DURANTE, L.; VIVES, P.; CLEFF, M. B. Eliminação de Dioctophyme renale pela urina em canino com dioctofimatose em rim esquerdo e cavidade abdominal - Primeiro relato no Rio Grande do Sul. Arquivo Brasileiro de Medicina Veterinária e Zootecnia. v.693 p.618-622, 2017.

SAPIN, C. F.; SILVA-MARIANO, L. C.; GRECCOCORREAA, L. Dioctofimatose renal bilateral e disseminada em cão. Pesquisa Veterinária Brasileira. v.37 p.1499-1504, 2017.

TRINDADE, M. A. C.; MACEDO, M. R. P.; MULLER, G. Dioctophyme renale (Nematoda: Dioctophymatidae) in Leopardus geoffroyi 
(Carnivora: Felidae) in the Neotropical region. Revista Brasileira de Parasitologia Veterinária. v.27 p.223225, 2018.

URQUHART, G. M.; ARMOUR, J.; DUNCAN, J. L.; DUNN, A. M.; JENNINGS, F. W. Parasitologia veterinária. Rio de Janeiro: Guanabara Koogan, 2.ed. p.285, 1998.
VENKATRAJAIAH, N.; KALBANDE, S. H.; RAO, G. V.; REDDY, V. C.; REDDY, S. H.; RAO, P. R.; BABU, K.; KEERTHI, A. Dioctophymatosis renalis in humans: first case report from India. The Journal of the Association Physicians of India. v.62 p.70-73, 2014. 